\title{
MOBILE RSVP
}

Towards Quality of Service (QoS) Guarantees in a Nomadic Internet-based Environment

\author{
Ali Mahmoodian', Günter Haring ${ }^{2}$ \\ Institute for Applied Computer Science and Information Systems, Department for Advanced \\ Computer Engineering, Vienna University \\ 'mahmoodian@csi.com, ${ }^{2}$ haring@ani.univie.ac.at
}

\begin{abstract}
In this paper we introduce an extended RSVP protocol called Mobile RSVP which support multimedia and real-time traffic in a Mobile IP based environment. Our proposed protocol is appropriate for those mobile nodes that can not a-priori determine their mobility behaviour. However, we have also analysed the effect of a deterministic mobility specification. We also describe the conceptual design of the reservation mechanism of Mobile RSVP and discuss the hand-over procedure. Efforts have been made to analyse the performance of Mobile RSVP and to assess its overhead and control traffic.
\end{abstract}

Keywords: Quality of Service (QoS), RSVP, and Mobile IP

\section{INTRODUCTION}

Current mobile and cellular networks and protocols are only capable of providing best-effort delivery of information. Consequently, they are not suitable for real-time and multimedia communications, which often require throughput and delay guarantees from the transport system.

Recent research and development activities have been focusing on QoS issues in the wireline Internet. Protocols such as RSVP provide simplex QoS guarantees based on the requirements of heterogeneous receivers by making hop-by-hop resource reservation along the communication path.

However, QoS guarantees in a nomadic Internet-based environment remains an emerging research issue. Recognizing the need of such a protocol, we took a theoretical approach towards resource reservation in a mobile cellular network and propose the concept of an extended RSVP 
protocol, called Mobile RSVP, which is capable of providing QoS guarantees to mobile connections in a Mobile IP-based environment.

Mobole RSVP maintains the reserved portion of the connection until a new connection is set up, which is capable of providing QoS guarantees. Our proposed protocol also performs progressive resource reservation in advance, while the mobile host is travelling from cell to cell.

This paper is organized as follows. Sections 2 and 3 give a brief introduction to Mobile IP and RSVP, respectively. In Section 4 we discuss general service models and introduce our Mobile RSVP protocol. In Sention 5 we outline the protocol operation of Mobile RSVP and analyze various parameters. Section 6 presents various methods to enhance the performance of our proposed protocol. Section 7 discusses some open issues. We conclude in Section 8 with some future directions of this work.

\section{INTRODUCING MOBILE IP}

Mobile IP ([3] and [6]) is a modification to IP that allows nodes to continue receiving datagrams regardless of any movements and changes to the location and attachment to the Internet. It solves the mobility problem by maintaining two addresses for each mobile node, one for locating the mobile computer, and the other for identifying a communication endpoint on the mobile computer.

Mobile IP consists of the following three related activities:

Agent discovery - Home agents and foreign agents advertise their availability on the corresponding (sub)network they provide service. Mobile nodes may either listen or solicit agent advertisement.

Registration - Mobile node receives a so-called care-of address from the foreign agent corresponding to its current position and informs its home accordingly. Home agent decides whether a registration is authorised. It creates an association between the static IP address and the mobile node's current point of attachment (mobility binding) and subsequently sends a corresponding reply to the mobile node (Figure la).

Tunnelling (Figure 1b) - Datagrams received by the home agent containing the static IP address of the mobile node are encapsulated and forwarded (tunnelled) to the mobile node's new point of attachment. Foreign agent decapsulates incoming datagrams and passes them to the mobile node. Triangle routing, however, is not optimal and may cause home agent bottleneck, network congestion and an increased sensitivity to network partitions. Performance can be improved by providing the correspondent 
node with the mobility binding update, which enables datagrams to be sent directly to the mobile node's care-of address.

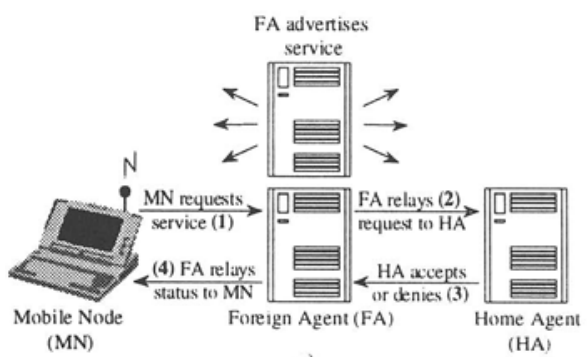

a)

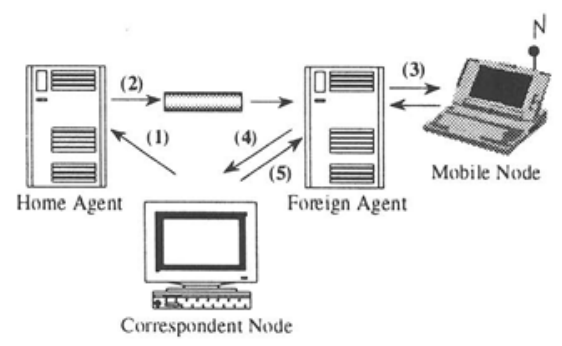

b)

Figure 1: a) Agent advertisement and the four-step registration process of Mobile IP. b) Tunnelling process of Mobile IP using route optimisation

\section{INTRODUCING RSVP}

Resource Reservation Protocol (RSVP) is a protocol specially designed for Integrated Services Internet. It enables applications to set up reservations over the network for various services required. RSVP is a receiver based model and hence enables heterogeneous receivers to make reservations specifically tailored to their own needs. The operation of RSVP can be divided as follows (Figure 2):

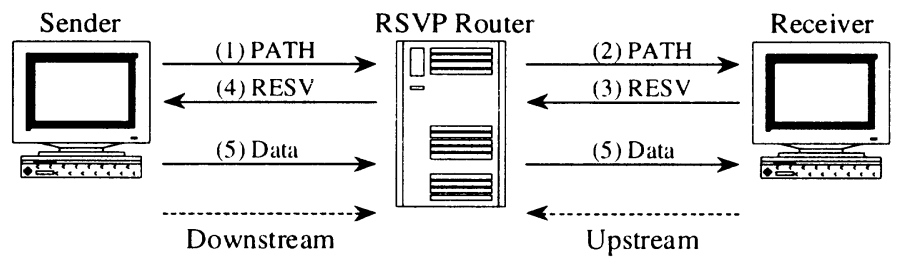

Figure 2: Operation of RSVP

(1) An RSVP sender that aims to initiate a session sends a PATH message to the corresponding receiver. The PATH message travels hop-byhop (downstream) through the network and passes subsequent RSVP routers installing "path state" in each node along the way, which includes at least the unicast IP address of the previous upstream router. The router may also update an ADSPEC object contained in the PATH message, which summarizes the path's characteristics. The ADSPEC object is delivered to 
the receiver to ensure a precise decision about how much bandwidth and delay to ask for. (2) Upon analyzing the obtained PATH message and performing necessary re-calculation of the traffic specification (ADSPEC), the PATH message is finally forwarded to the receiver. (3) The receiver evaluates the PATH message and generates a RESV message, which is eventually sent to the network. (4) The generated RESV message passes the routers in the reverse order (upstream), creates, and maintains "reservation state" in each node along the path. (5) Upon receiving the RESV message, the sender initializes appropriate traffic control parameters at the first hop (the sender) and starts transferring data.

To make best use of the reserved state in the network, RSVP supports various reservation styles and merging of similar reservations ([5] and [7]).

\section{MOBILE RSVP (A PROPOSAL)}

Anup K. Talukdar et al. ([4]) refers to two classes of real-time applications: tolerant applications, which can adapt to packet delays, and intolerant applications, which cannot tolerate any delay bound violations. Correspondingly, two different service models have been defined: guaranteed service for intolerant applications, and predictive service for tolerant applications. Furthermore, three service classes are proposed to which mobile nodes may subscribe.

Unfortunately, all approaches assume that the mobility of a user is predictable so that mobility can be characterized precisely by the Mobility Specification (Mobility Profile). Consequently, a mobile node is required to negotiate the desired QoS at the beginning of the data flow session. However, mobile node might not have an a-priori knowledge of its future mobility behaviour.

In our proposed reservation protocol, in particular, we have considered non-deterministic mobility behaviour. Hence, our proposed protocol will enable a higher degree of flexibility for those mobile nodes, which might not be able to adhere to a pre-defined mobility specification.

Based on the above specification, we have proposed the Mobile RSVP protocol, which is an extension of RSVP standardised by IETF for reserving resources between Internet nodes. Mobile RSVP works with Mobile IP; consequently, the assumption is made that Mobile IP is known throughout the network.

We assume that route optimisation is employed in the underlying Mobile IP protocol to facilitate the correspondent node with an up-to-date mobility binding. It is further assumed that each cell is connected to the fixed 
network through a base station. In the proposed network architecture, base stations are considered as part of Mobile IP routers with the facility to communicate with and keep track of mobile nodes. Preferably, there should be a wired direct connection between neighbouring base stations. Our proposed network is divided into microcellular or picocellular sub-networks capable of supporting both data and multimedia communication, whereby the bandwidth shall be divided into two portions: one for time constrained traffic on a reservation basis; and the other for connectionless non-timeconstrained traffic on a contention basis, basically for control information. Each base station co-ordinates and administers channel access and bandwidth reservation in a cell.

Mobile RSVP extends the functionality of PATH and RESV message to also perform passive reservation of resources and introduces two major new messages ACTIVATE and UPDATE to activate passive reservations and to update previous reservations in case of service degradation.

\section{PROTOCOL OPERATION}

We assume that a mobility binding already exists to create an association between a home agent and the corresponding care-of address, along with the remaining lifetime of the association. Thus, datagrams can be tunnelled to the foreign agent and subsequently forwarded to the mobile node.

For simplicity, we consider a simplex unicast communication link between sender and receiver.

\subsection{Mobile RSVP Connection Set-up}

To avoid reservation of resources in the triangle route, a four-way handshake is proposed:

1. A traffic source that aims to initiate a Mobile RSVP session sends a CONreq message.

2. A receiver willing to obtain (multimedia) data from the traffic source responds via a CONconf message. The latter message contains the current care-of address of the mobile node as a parameter. Hence, the correspondent node is informed of the mobile node's current location. A CONrej is generated in case the receiver is not able or willing to be involved in a communication session with the traffic source. 
3. The sender sends a PATH message directly to the mobile node's care-of address, which travels hop-by-hop (downstream) through subsequent RSVP routers installing (passive) QoS state in each router along the path.

4. Mobile node analyses the obtained PATH messages and generates a RESV message accordingly, which passes the routers in the reverse order (upstream). Each RSVP router has to evaluate the RESV message and make appropriate reservation.

\subsection{Progressive Resource Registration}

Since the mobile node is roaming, each foreign agent has to make sure that resources are available as the relevant mobile node moves to the neighbouring networks. Therefore, a modified reservation model has to be performed, which is referred to as Progressive Resource Registration.

Based on the latter reservation model, a foreign agent acts as a traffic source and distributes PATH messages to the neighbouring base stations. PATH messages advise the neighbouring base stations of the characteristics of the sender traffic and install path state in corresponding nodes. If $R_{\max }$ is the maximum resource contingent that can be allocated in a router (based on the Fluid Model and the corresponding Leaky-Bucket mechanism discussed in [8]) at a particular point of time and $R_{u}$ is the resource requirement announced by the previous (upstream) router, then resources can be allocated only if $R_{a} \leq R_{\max }$. We can distinguish between three different cases:

1. $R_{a} \leq R_{\max }$ : The corresponding request is forwarded to the next hop.

2. $R_{a}>R_{\text {max }}: R_{a}^{\prime}$ is calculated based on available resources. PATH is forwarded to the next hop using $R_{a}^{\prime}$ as the modified parameter.

3. The request can not be registered: Using a REJECT message, the PATH originating node (current foreign agent) is notified that the requested reservation can not be registered. The complete branch is then considered as a best-effort connection.

Each base station itself has finally to analyse the reservation request and either confirm or reject the request. In case of a confirmation, RESV messages are sent to the current foreign agent from each individual neighbouring base station (Figure 3a). Every RESV message makes appropriate passive reservation along its corresponding branch.

A reservation requires an entry in a database, such as $\left\langle\right.$ SessionID, $\left.R_{a} \bullet, t_{\text {life }}\right\rangle$, where $t_{\text {life }}$ indicates the lifetime of the registration, after which it will be deleted. The node, which has initiated the reservation, is automatically assigned with the highest priority for his required resources. On one hand, this prioritisation ensures the mobile node that its reserved resources are available as soon as it enters the corresponding cell. On the other hand, it 
enables other mobile nodes to consume reserved but still unused (passive) resources. It is obvious that registered resources have to become available as soon as the mobile node with the highest priority requests them. Thus, the resource allocation is pre-emptive. A foreign agent, who wishes to maintain the registration of its reservation request, has to refresh $t_{\text {life }}$, periodically. The introduction of $t_{\text {life }}$ will prevent the blocking of other requests being registered.

The potential reservation state of each individual path to the neighbouring base station along with the corresponding branch identification is maintained by the foreign agent. This information will be of use during hand-over.

\subsection{Hand-over Procedure in Mobile RSVP}

The hand-over procedure of Mobile RSVP is based on the Mobile IP protocol. It assumes that a mobile node is successfully handed over to the adjacent base station and that the home agent has been notified, accordingly.

\subsubsection{Activating Passive Reservation in the New Environment}

After the mobile node has changed its point of attachment, the previous foreign agent has also to hand-over QoS requirements to the new foreign agent.

According to the Mobile IP protocol, the former foreign agent has to keep record of the new care-of address of the mobile node for those packets that have been sent to the old care-of address while the mobile node was changing its point of attachment. keeps this information until an appropriate link is established between the correspondent node and the mobile node. Mobile RSVP also suggests activating adequate resources between two neighbouring base stations:

To change the state of a reservation from passive to active, the former foreign agent $F A_{I}$ in the cell $C_{I}$ (Figure $3 \mathrm{~b}$ ) has to send an ACTIVATE message to the corresponding agent in the neighbouring cell $C_{2}\left(F A_{2}\right)$ along the same path, which has been established during the passive reservation. The correct path is identified through the relevant session identification. In case of a service degradation, which is determined by comparing the reservation state of the selected path (path to $F A_{2}$ ) with the current service specification, $F A_{l}$ has to inform all previous nodes involved in the session (including the traffic sender) and update their reservation states accordingly. This action is performed using an UPDATE message sent in the reverse path from the current foreign agent to the sender. 


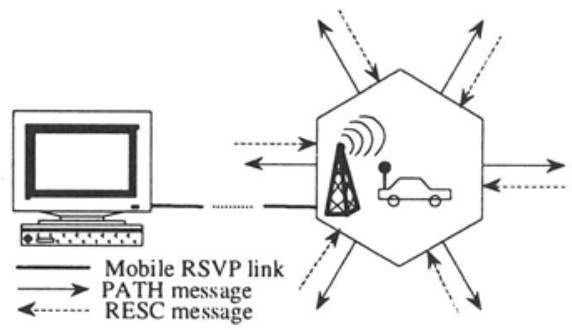

a)

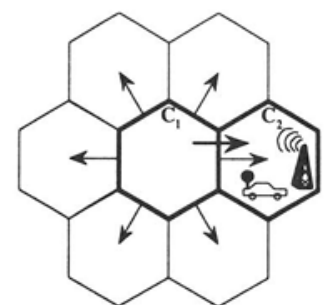

c)

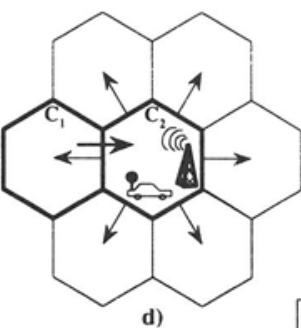

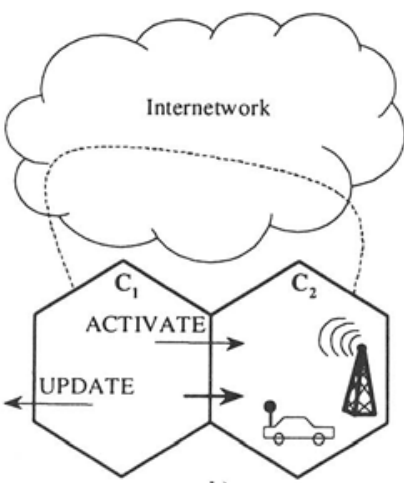

b)

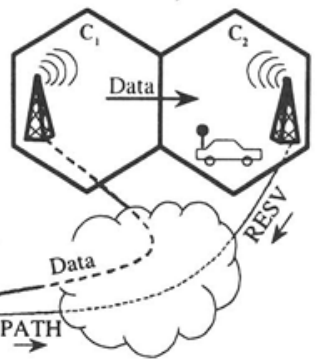

Figure 3: Operation of Mobile RSVP.

\subsubsection{Release and Record Resource Reservations}

When a mobile node moves to a new point of attachment, passive reservations in the old neighbourhood become obsolete. Hence, either they time out or they have to be terminated or "released". For this reason, the old foreign agent distributes a RELEASE message to its neighbourhood (Figure $3 c$ ) informing them that the relevant registration is not valid any longer.

On the other hand, the new foreign agent is responsible to pre-advice (PATH message) its neighbourhood with regard to the arriving mobile node (Figure 3d). This will again initialise the Progressive Resource Registration and generate reservation requests.

\subsubsection{New Mobile RSVP Link to the Current Care-of Address}

During the process of roaming, a mobile node has to ensure that at least two databases are updated. Firstly, a database maintained by the home agent, which contains mobility bindings of native nodes that are currently travelling $(\langle$ StaticIP, care_of address, lifetime $\rangle)$ and secondly, a database 
maintained by correspondent nodes reflecting the mobile node's current point of attachment. When a correspondent node capable of providing Mobile RSVP services receives an updated mobility binding, it automatically initiates a new Mobile RSVP link to mobile node's new point of attachment by sending a PATH message to the mobile node (Figure $3 \mathrm{e}$ ). However, establishing a new RSVP link is a matter of trade-off and has to be decided by the RSVP initiating entity.

At the same time, multimedia datagrams directed towards the mobile node are forwarded through the old foreign agent $F A_{l}$ to the new destination $F A_{2}$ of the mobile node along the Mobile RSVP connection between $F A_{l}$ and $F A_{2}$. Upon receiving the PATH message, $F A_{2}$ initiates a reservation by sending a correspondent RESV message. When the correspondent node receives the RESV message it automatically updates the mobility binding and starts sending datagrams to the $F A_{2}$, probably at a modified service quality. This also shows the flexibility of Mobile RSVP to adjust to variable network conditions.

The so-called "post-hand-over" procedure is realised in the Mobile RSVP layer of the corresponding protocol stack.

It is the responsibility of the Mobile RSVP instance to temporarily store updated mobility bindings until a new communication link with guaranteed QoS becomes available. Upon termination of a session, which is initiated by an END message, corresponding registrations are released based on the instruction of the relevant foreign agent.

\subsection{Protocol Analysis}

In this section, we try to calculate the response time of call set-up, connection hand-over and resource registration for a unicast mobile connection between a mobile node and a correspondent node. We also try to assess the amount of control traffic generated by Mobile RSVP.

The following parameters are used:

$H_{a} \quad$ Avg. number of hops along an active Mobile RSVP connection

$H_{c h} \quad$ Avg. number of hops between home agent and correspondent node

$H_{h f} \quad$ Avg. number of hops between home agent and foreign agent

$H_{\text {cell }} \quad$ Avg. number of hops between two foreign agents in neighbouring cells

$N_{\text {neighbour }}$ Number of neighbouring cells

$\tau_{w} \quad$ Avg. transmission delay over a wired link

$\tau_{w l} \quad$ Avg. transmission delay over a wireless link

$\tau_{r} \quad$ Avg. routing delay in a router or base station

$\tau_{\text {rd }} \quad$ Avg. reservation processing delay in a router or base station

$\tau_{\text {caps }} \quad$ Avg. encapsulation or decapsulation delay of datagrams 


\subsubsection{Response Time during Mobile RSVP connection set-up}

Let $t_{s}$ be the time it takes to establish a mobile connection, measured from the moment the mobile host makes the request until its acceptance. It is given by $t_{s}=t_{1}+4 \tau_{w /}+H_{a}\left(3 \tau_{w}+2 \tau_{r}+\tau_{r d}\right)$, whereby $t_{1}=2 \tau_{\text {caps }}+\tau_{w} \tau_{r}\left(H_{c h}+H_{h f}\right)$, which includes the time for encapsulation and decapsulation of the CONreq message at both ends of the tunnel and the time to transmit and route the initial message from the correspondent node to the mobile node's current point of attachment.

\subsubsection{Response Time during Progressive Resource Registration}

Let $t_{p}$ be the time it takes to process a Progressive Resource Registration measured from the moment the current foreign agent initiates the event until its eventual establishment. It is given by $t_{p}=N_{\text {neikhthour }} H_{c e l l}\left(2 \tau_{w}+\tau_{r}+\tau_{r d}\right)$.

\subsubsection{Response Time during Mobile RSVP hand-over ${ }^{1}$}

Let $t_{h}$ express the hand-over latency. If the mobile node is transmitting or receiving when it crosses cell boundary, then $t_{h}$ is measured from the moment the mobile node greets the new foreign agent until it can resume transmission or the switchover occurs at the crossover node. It is given by $t_{h}=\tau_{w}\left(\tau_{r d} H_{a}+H_{c e l l}\right)+t_{n}$, where $t_{n}$ refers to the time it takes to establish a new Mobile RSVP link to the mobile node's new point of attachment, to release registered resources and to perform appropriate Progressive Resource Registration given by $t_{n}=t_{p}+H_{\text {cell }} \tau_{w}\left(N_{\text {neighthur }}-1\right)+t_{\text {new }}$, whereby $t_{\text {new }}=H_{a}\left(2 \tau_{w}+\tau_{r}+\tau_{r d}\right)+2 t_{w \prime}$ and refers to the time to establish a new Mobile RSVP link from the correspondent node to mobile node's new point of attachment.

\subsubsection{Protocol Overhead and Control Traffic}

Classified in Table 1 are the protocol messages (including acknowledgements) generated in the events of connection establishment, Mobile RSVP hand-over and connection termination. In general, the amount

\footnotetext{
'It should be noted that the response time of the Mobile IP handoff procedure is not included in our calculation.
} 
of control traffic is linearly proportional to the number of neighbouring cells $N_{\text {neythour }}$.

Our proposed protocol generates even less control traffic when the reservation is initiated by the mobile node, i.e. the mobile node sends a PATH message to the corespondent node, provided the correspondent node is connected to a fixed ("wired") network. The a-priori knowledge of the correspondent node's address makes initial set-up messages CONreq and CONconf superfluous, since the mobile node does not need to go through the home agent to reach a correspondent node. In this case, the total number of control messages will reduce by four.

\begin{tabular}{|c|c|c|c|c|}
\hline & $\mathbf{M N} \leftrightarrow \mathbf{F A}$ & $\begin{array}{l}\text { FA↔fixed } \\
\text { network }\end{array}$ & $\mathbf{F A} \leftrightarrow \mathbf{F A}$ & Total Number \\
\hline $\begin{array}{l}\text { Connection } \\
\text { set-up }\end{array}$ & $\begin{array}{l}1 \times \text { CONreq } \\
1 \times \text { CONconf } \\
1 \times \text { PATH } \\
1 \times \text { RESV }\end{array}$ & $\begin{array}{l}1 \times \text { CONreq } \\
1 \times \text { CONconf } \\
1 \times \text { PATH } \\
1 \times \text { RESV }\end{array}$ & 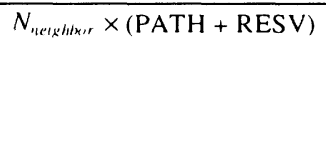 & $2 N_{\text {neryther }}+8$ \\
\hline $\begin{array}{l}\text { Mobile RSVP } \\
\text { hand-over }\end{array}$ & & $1 \times$ UPDATE & $\begin{array}{l}1 \times \text { ACTIVATE } \\
N_{\text {nely } h h_{k, r}} \times \text { RELEASE } \\
N_{\text {netgh } h \text { rer }} \times(\text { PATH }+ \text { RESV })\end{array}$ & $3 N_{\text {neightor }}+2$ \\
\hline $\begin{array}{l}\text { Post hand- } \\
\text { over }\end{array}$ & $\begin{array}{l}1 \times \text { PATH } \\
1 \times \text { RESV }\end{array}$ & $\begin{array}{l}1 \times \text { PATH } \\
1 \times \text { RESV }\end{array}$ & $N_{\text {neighlkx, }} \times(\mathrm{PATH}+\mathrm{RESV})$ & $2 N_{\text {neighhor }}+4$ \\
\hline $\begin{array}{l}\text { Connection } \\
\text { termination }\end{array}$ & $1 \times$ END & $1 \times \mathrm{END}$ & $N_{\text {neighlk,r }} \times$ RELEASE & $N_{\text {neighthor }}+2$ \\
\hline
\end{tabular}

Table 1: Control messages in the Mobile RSVP

\subsection{Performance Optimisation of Mobile RSVP}

The main goal of performance optimisation in Mobile RSVP is to reduce control traffic and protocol overhead. As we have seen, the amount of control traffic increases linearly proportional to the number of neighbouring cells $N_{\text {neighbour }}$. Hence, one major source of improvement is to reduce the number of communicating neighbours. One may take the mobility behaviour and the trajectories of mobile node into account. For example, it is quite unlikely that a mobile node return to the cell that it just left. A foreign agent can also determine the direction of motion of mobile nodes from the sequence PATH messages associated with it (Figure 4a).

This information can be used by the foreign agent to decide whether to proceed Progressive Reservation Registrations, or it can be used by the 
network to adapt algorithms such as the Adaptive Prioritised Link Partitioning proposed by [2]. The result would be less control overhead and better network efficiency.

Another approach would be (and this applies also to the policy control modul of RSVP) the implementation of a priority mechanism that allows users to send reservation requests with higher priority than others. The Integrated Services (IS) may be coupled with a billing system that charges the user according to priority level of his reservation request.

An a-priori knowledge of the mobile node's mobility behaviour, where the corresponding foreign agents are pre-determined might help to reduce control traffic and improve service availability. In fact, for this kind of applications Mobile RSVP does not represent an appropriate reservation model. In such cases, it is more convenient to perform resource reservation in a timely manner, where QoS can be guaranteed starting at a particular point of time and lasting for a certain period.

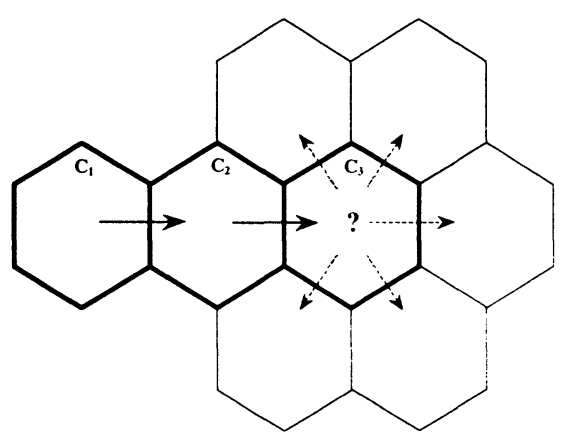

a)

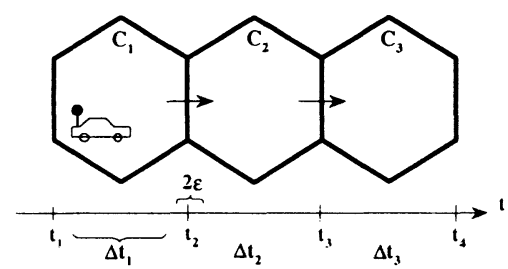

b)

Figure 4: a) Assessing the mobility behaviour of the mobile node based on the previous motion path. b) Resource reservation model for mobile nodes with deterministic behaviour.

Hence, reservation has to be made in advance when the mobile node decides to leave its home network, so each foreign agent knows approximately when a mobile node will arrive in the respective cell (network) and how long it plans to stay. Foreign agents might be notified of any potential delay that might occur. This reservation model is best applicable if the mobile node is travelling for example by car or train, where the mobility route is somehow pre-determined. However, it does not provide the flexibility a mobile node might require, but is exemplifies an equitable approach for a certain class of mobility behaviour. 
Figure $4 \mathrm{~b}$ illustrates such a reservation model. In a deterministic mobility behaviour model the equation $t_{\text {tout }, ~}=\Delta t_{i}+2 \varepsilon$ should hold, where $t_{\text {totol, }, i}$ is total time a reservation should be maintained in the corresponding cell $i$ for a specified mobile node and $\Delta t_{i}=t_{t+1}-t_{t}$, which is the time the mobile node will occupy cell $i$. $\varepsilon$ refers to the average time required for hand-over. $2 \varepsilon$ is required to enable a smooth hand-over and depends upon the corresponding hand-over algorithm.

\subsection{Open Issues}

A major problem of Mobile RSVP will be its applicability in the current network infrastructure. It is required that relevant routers of the underlying network are aware of Mobile RSVP, otherwise the control traffic will be very high and will even affect the best-effort performance. As a first approach, it is suggested that Mobile RSVP be realised in corporate Intranets to provide multimedia or other real-time data to mobile nodes.

Another pitfall of Mobile RSVP is its control traffic and protocol overhead, which increases rapidly when both communication counterparts are mobile hosts. In case the correspondent node is mobile himself, a huge amount of additional control messages is necessary, which would make Mobile RSVP quite inefficient, since the underlying network structure might get overloaded with required protocol overhead.

In this case, both involved parties (mobile hosts) have to employ progressive resource registration. This will extremely increase the complexity of a potential mobile resource reservation protocol.

In a multicast scenario, the control traffic will be even higher. Appropriate measures have to be considered to overcome this pitfall. The concept of Differentiated Services ([8] and [9]) might help in this respect to reduce control traffic. This approach will remain for further investigation and research.

\subsection{Concluding Remarks}

Motivated by the challenge of providing QoS guarantees to mobile users, an extended RSVP protocol (Mobile RSVP) has been proposed, which could support multimedia and real-time traffic in a Mobile IP based environment. Mobile RSVP is appropriate for those mobile nodes, which can not a-priori determine their mobility behaviour. In addition, the effect of a deterministic mobility specification has been analysed and proposals for more appropriate protocols have been outlined. 
In addition, we have described the conceptual design of the reservation mechanism of Mobile RSVP and discussed the hand-over procedure. Efforts have been made to analyse the performance of Mobile RSVP and to assess its overhead and control traffic.

As far as future work is concerned, the focus will be at simulating the behaviour of Mobile RSVP under various network conditions based on a performance model for Mobile IP networks. It is also necessary to evaluate, experiment and propose mechanisms for assessing mobility behaviour, which would eventually lead to the definition of a mobility profile.

We have also started to investigate and research a liaison between Mobile IPv6 and RSVP, since IPv6 provides more sophisticated flow control and QoS provision mechanisms. Furthermore, mobility support concepts have already been drafted and proposed in [1].

\section{References}

[1] David B. Johnson and Charles E. Perkins, "Mobility Support in IPv6", Internet Draft, draft-ietf-mobileip-ipv6-03.txt, July 30, 1997.

[2] Kam Lee, "Supporting mobile multimedia in integrated services networks", Wireless Networks, Vol. 2, pp. 205-217, 1996.

[3] Charles E. Perkins, "Mobile IP, Adding Mobility to the Internet", MobiCom'97, The Third Annual ACM/IEEE International Conference on Mobile Computing and Networking, September 26, 1997.

[4] Anup K. Talukdar, B. R. Badrinath and Arup Acharya, "On Accomodating Mobile Hosts in an Integrated Services Packet Network", IEEE Proceedings of the Infocom 97, April 1997, pp. 10641053.

[5] Paul P. White, "RSVP and Integrated Services in the Internet: A Tutorial", IEEE Communications Magazine, May 1997, pp. 100-106.

[6] Charles E. Perkins and David B. Johnson, "Route Optimization in Mobile IP", Internet Draft, available at draft-ietf-mobileip-optim06.txt, July 29, 1997.

[7] R. Braden, L. Zhang, S. Berson, S. Herzog and S. Jamin, "Resource ReSerVation Protocol (RSVP), Version 1 Functional Specification", $R F C 2205$, available at $\mathrm{ftp}: / / \mathrm{ds}$.internic.net/rfc/rfc2205.txt.

[8] C. Metz, "IP QoS: Travelling in First Class on the Internet", Internet Computing, Vol. 3, Nr. 2, pp. 84-88, March/April 1999.

[9] S. Blake et al., "An Architecture for Differentiated Services", RFC 2475, available at $\mathrm{ftp} / / / \mathrm{ds}$.internic.net/rfc/rfc2475.txt. 